\title{
Relationship between Respiratory Muscle Strength and Exercise Tolerance
}

\author{
HiROMICHI SUGIURA, RPT, MS ${ }^{1)}$, KIYOHITO OHTA, RPT, MS ${ }^{2)}$, SATSUKI MinATANI, RPT ${ }^{2)}$, \\ HIRONORI TANOUE, RPT ${ }^{2}$, AKIRA KOKUBO, RPT, MS ${ }^{3)}$, YOSHIKIYO KANADA, RPT, $\mathrm{PhD}^{4}$, \\ SHUNJI SAKO, RPT, MS ${ }^{2)}$ \\ ${ }^{1)}$ Department of Physical Therapy, Faculty of Health Science, Suzuka University of Medical \\ Science: 1001-1, Kishioka-cho, Suzuka, Mie 510-0293, Japan. TEL: +81 59-383-8991, \\ FAX: +81 59-383-9666, E-mail: sugiura@suzuka-u.ac.jp \\ ${ }^{2)}$ Department of Rehabilitation, Major in Physical Therapy, Gifu Junior College of Health \\ Science \\ ${ }^{3)}$ Department of Physical Therapy, Nagoya Collage of Rehabilitation Medicine and Social \\ Welfare \\ 4) Faculty of Rehabilitation, School of Health Sciences, Fujita Health University
}

\begin{abstract}
Purpose] This study was performed to clarify the relationship between respiratory muscle strength and exercise tolerance. [Subjects and Methods] Nine healthy adult male subjects (mean age 20.9 \pm 0.3 years) were instructed to perform a ramp load exercise using a cycle ergometer. As measures of inspiratory muscle strength, maximal expiratory mouth pressure (PEmax) and maximal inspiratory mouth pressure (PImax) were measured before exercise and immediately, 15 minutes and 30 minutes after exercise. As measures of respiratory response, minute ventilation, respiratory frequency, tidal volume and oxygen uptake were measured using an exhaled gas analyzer. Heart rate was calculated from R-R intervals on an electrocardiogram as a measure of heart rate response. [Results] The results revealed significant correlations between PEmax and maximal exercise load, PImax and maximal exercise load, PEmax and peak $\dot{\mathrm{V}} \mathrm{O}_{2}$ and PImax and peak $\dot{\mathrm{V}} \mathrm{O}_{2}$. PEmax measured immediately after exercise was lower than that measured immediately before exercise. [Conclusion] These findings suggest a correlation between respiratory muscle strength, especially expiratory muscle strength, and exercise performance.
\end{abstract}

Key words: Maximal mouth pressure, Maximal exercise load, peak $\dot{\mathrm{V}}_{2}$

(This article was submitted Jun. 24, 2009, and was accepted Aug. 17, 2009)

\section{INTRODUCTION}

At rest, inspiratory muscle activity is dominant with little or no expiratory muscle activity. During exercise, breathing is hyperventilatory and requires expiratory muscle activity as well as an increase in inspiratory muscle activity ${ }^{1-3}$. Respiratory muscle activity plays an important role in ventilation control and is especially essential for respiratory response during exercise.

Previous studies have shown that reducing the load on respiratory muscles with the use of helium mixed gas resulted in relief of the sense of dyspnea and improvement in exercise tolerance in patients with chronic obstruction pulmonary disease ${ }^{4,5)}$ and chronic heart failure $\left.{ }^{6}\right)$ who were instructed to perform exercise. This improvement in exercise tolerance induced by a reduced load on the respiratory muscles suggests a correlation between respiratory muscle strength and exercise performance and an important role for respiratory muscle strength in the maintenance and exertion of 
exercise performance. However, the nature of the relationship between respiratory muscles and exercise performance is unclear and expiratory and inspiratory muscles have not yet been investigated separately. Thus, the present study aimed to clarify this relationship by determining the relationships of expiratory and inspiratory muscle strength with exercise tolerance.

\section{SUBJECTS AND METHODS}

The subjects were 9 healthy adult men (mean age $20.9 \pm 0.3$ years, mean body height $170.9 \pm 2.8 \mathrm{~cm}$, mean body weight $64.5 \pm 5.7 \mathrm{~kg}$ ), who did not smoke and were not athletes. We confirmed that none of the subjects had suffered any respiratory or cardiovascular disease within the past 1 month. All subjects were given explanations about the details and risks of the study and asked to sign a consent form prior to participation in the study.

The following body composition parameters were measured before starting exercise using a body composition meter (BC-118E, Tanita, Tokyo, Japan): body weight, percent body fat, lean body weight, skeletal muscle mass, and body mass index (BMI).

The following pulmonary function parameters were measured using a spirometer (AS-507, Minato, Osaka, Japan): forced vital capacity (FVC), forced expiratory volume in one second $\left(\mathrm{FEV}_{1}\right)$, and percent of forced expiratory volume $\left(\mathrm{FEV}_{1.0 \%}\right)$, and as measures of respiratory muscle strength, maximal inspiratory mouth pressure (PImax) and maximal expiratory mouth pressure (PEmax). Subjects were instructed to hold the cheeks with the hands to prevent air leakage and to keep the trunk stable during measurement. At the start of the measurement, subjects were instructed to maintain maximal mouth pressure for $1.5 \mathrm{~s}$ at the maximal expiratory level to measure PImax and at the maximal inspiratory level to measure PEmax. The measurements were repeated 10 times. The three highest measurement values with an error of $5 \%$ or less were selected and the mean of the three values was calculated ${ }^{7)}$. As measures of respiratory response, minute ventilation $\left(\dot{\mathrm{V}}_{\mathrm{E}}\right)$, respiratory frequency ( $\mathrm{f})$, tidal volume $\left(\dot{\mathrm{V}}_{\mathrm{T}}\right)$ and oxygen uptake $\left(\mathrm{VO}_{2}\right)$ were measured using an exhaled gas analyzer (AE-300S, Minato, Osaka, Japan) on a breath-by-breath basis. Heart rate (HR) was also calculated from R-R intervals on an electrocardiogram (ECG-9522, Nihon Kohden, Tokyo, Japan) as a measure of heart rate response. For the assessment of the maximal respiratory and heart rate responses during exercise, peak $\dot{\mathrm{VO}}_{2}$ was calculated as the mean value during the 30 -s period before the load reached the maximum; other measures were also calculated in the same fashion.

Subjects were instructed to perform cycle exercise using a cycle ergometer (75XL II ME, Combi Wellness, Tokyo, Japan) with the ramp load set at $20 \mathrm{~W} / \mathrm{min}$ and pedal rotation rate at 55-60 rpm. The exercise was completed when the pedal rotation rate fell below $55 \mathrm{rpm}$.

Prior to the start of the study, subjects were familiarized with the procedure of the study and instructed to perform 10-minute cycle exercise at a constant load of $20 \mathrm{~W}$ (warm-up). Body composition parameters were measured first, followed by pulmonary function parameters and maximal mouth pressures. Subjects were instructed to stay at rest for 1 minute on the cycle ergometer before starting the ramp load exercise. After finishing the load exercise, subjects were required to continue the cycle exercise without load for 3 minutes as a cool-down. Pulmonary function parameters and maximal mouth pressure were then measured immediately, 15 minutes and 30 minutes after exercise completion.

To examine the effect of exercise on pulmonary function and mouth pressure, pulmonary function parameters and maximal mouth pressures were compared before and after exercise using Friedman's $\chi^{2}$ r-test. Significant differences $(p<0.05)$ were further analyzed with Dunnett's test and Wilcoxon's t-test. In addition, to examine the relationship of respiratory muscle strength to maximal exercise load and respiratory response, the correlations of pre-exercise maximal mouth pressures to maximal exercise load and respiratory response parameters $\left(\dot{\mathrm{V}}_{\mathrm{E}}\right.$ and peak $\left.\dot{\mathrm{V}}_{2}\right)$ were determined. To examine the possible effect of skeletal muscle mass on maximal exercise load and peak $\dot{\mathrm{V}} \mathrm{O}_{2}$, the correlations of skeletal muscle mass to maximal exercise load and peak $\mathrm{VO}_{2}$ were also determined. Correlations were determined using Spearman's rank correlation coefficient. All statistical analyses were performed using statistical analysis software, SPSS for Windows (version 16.0.2J). 
Table 1. Body composition of subjects

\begin{tabular}{lll}
\hline Body weight & $(\mathrm{kg})$ & $64.5 \pm 2.7$ \\
Body fat & $(\%)$ & $14.1 \pm 2.9$ \\
Lean body weight & $(\mathrm{kg})$ & $55.3 \pm 4.1$ \\
Skeletal muscle mass & $(\mathrm{kg})$ & $52.4 \pm 3.9$ \\
BMI & $\left(\mathrm{kg} \cdot \mathrm{m}^{-2}\right)$ & $22.0 \pm 1.8$ \\
\hline
\end{tabular}

Mean \pm SD

\section{RESULTS}

The means and standard deviations (SD) of the body composition parameters examined are summarized in Table 1.

Regarding post-exercise pulmonary function, no significant differences from baseline were found in FVC and FEV measured immediately, 15 minutes and 30 minutes after exercise, while $\mathrm{FEV}_{1.0 \%}$ measured immediately after exercise was significantly higher than at the baseline. With regard to post-exercise maximal mouth pressure, a significant decrease in PEmax from baseline was observed immediately after exercise, but not at 15 and 30 minutes after exercise. No significant changes from baseline were observed for PImax measured immediately, 15 minutes and 30 minutes after exercise (Table 2). The mean \pm SD of maximal exercise load was $3.1 \pm 0.3 \mathrm{~W} / \mathrm{kg}$ and that of peak $\dot{\mathrm{VO}}_{2}$ was $39.1 \pm 3.6 \mathrm{ml} / \mathrm{min} / \mathrm{kg}$. Similarly, those of respiratory and cardiovascular response parameters on maximal exercise load were as follows: $\dot{\mathrm{V}}_{\mathrm{E}} 87.3 \pm 11.4 \mathrm{l} / \mathrm{min}, \mathrm{f} 44.6 \pm 9.8$ beats/ $\min , \dot{\mathrm{V}}_{\mathrm{T}} 2.0 \pm 0.3 \mathrm{l} / \mathrm{min}$ and HR $191.1 \pm 10.7$ beats/ min. Significant correlations were observed between PEmax and maximal exercise load $(\mathrm{r}=0.82$, $\mathrm{p}<0.01)$ and PEmax and peak $\dot{\mathrm{VO}}_{2}(\mathrm{r}=0.66, \mathrm{p}<0.05)$, whereas no significant correlations were observed between PImax and maximal exercise load $(\mathrm{r}=0.52$, $\mathrm{p}=0.15)$ and PImax and peak $\dot{\mathrm{V}}_{2}(\mathrm{r}=0.57, \mathrm{p}=0.10)$. No significant correlations were observed between PEmax and $\dot{\mathrm{V}}_{\mathrm{E}}(\mathrm{r}=0.22, \mathrm{p}=0.57)$, PImax and $\dot{\mathrm{V}}_{\mathrm{E}}$ $(\mathrm{r}=0.52, \mathrm{p}=0.15)$, and skeletal muscle mass and maximal exercise load $(\mathrm{r}=0.13, \mathrm{p}=0.79)$ or between skeletal muscle mass and peak $\dot{\mathrm{VO}}_{2}(\mathrm{r}=0.10$, $\mathrm{p}=0.99$ ).

\section{DISCUSSION}

The present study was performed to clarify the relationship between respiratory muscle strength and exercise tolerance.
The results revealed correlations of PEmax to maximal exercise load and peak $\dot{\mathrm{V}} \mathrm{O}_{2}$, with a particularly strong correlation found between PEmax and maximal exercise load. PEmax measured immediately after exercise was lower than that measured immediately before exercise. These findings suggest a correlation between respiratory muscle strength, especially expiratory muscle strength, and exercise tolerance.

Regarding the present method of producing exercise load, subjects were instructed not to make a compensatory body motion, such as forward/ backward and left/right motion of the body trunk, during cycling exercise at the maximal load, in order to precisely measure the exercise performance of the legs. Therefore, pedal rotation frequency was more prone to decrease compared with performance of regular ramp load exercise, which might have led to the absence of maximal oxygen uptake and maximal breathing capacity before the end of exercise. A possible exercise-limiting factor is skeletal muscle mass; however, there was no correlation between skeletal muscle mass and maximal exercise load. Another possible limiting factor was increased load on the respiratory muscles due to increased ventilatory response. Increased load on the respiratory muscles during exercise has been shown to increase the sense of dyspnea and possibly reduce output stimuli from the motor center to muscles ${ }^{8-10)}$. This suggests that, in response to increased load on the respiratory muscles due to increased ventilatory response, respiratory muscle strength maintains a high level of exercise performance even during high-intensity exercise.

The positive correlation between PEmax, maximal exercise load and maximal oxygen uptake observed in this study suggests that expiratory muscle strength is more closely related to exercise performance than inspiratory muscle strength. PEmax measured immediately after exercise was significantly lower than baseline PEmax, while no significant difference from baseline was found in FVC measured immediately after exercise. The lung volume, which is thought to be influenced by respiratory muscle strength, was therefore unchanged, whereas PEmax was decreased, indicating expiratory muscle fatigue. Expiratory muscles have a lower oxidative capacity and thus are known to be more prone to fatigue than inspiratory muscles ${ }^{11,12)}$. It has also been shown 
Table 2. Changes in pulmonary function, maximal mouth pressures after exercise

\begin{tabular}{cccccc}
\hline & Baseline & \multicolumn{3}{c}{ Post-exercise } \\
\cline { 3 - 5 } & & Immediately & 15 min & 30 min \\
\hline \multicolumn{2}{c}{} & & & & \\
pulmonary function & & & & & \\
FVC & $(l)$ & $4.62 \pm 0.43$ & $4.65 \pm 0.43$ & $4.67 \pm 0.48$ \\
FEV $_{1}$ & $(l)$ & $4.16 \pm 0.33$ & $4.18 \pm 0.30$ & $4.11 \pm 0.31$ & $4.13 \pm 0.33$ \\
FEV $_{1.0 \%}(\%)$ & $88.0 \pm 5.61$ & $90.56 \pm 6.56^{*}$ & $88.67 \pm 7.09$ & $89.0 \pm 6.96$ \\
maximal mouth pressures & & & & \\
PEmax & $\left(\mathrm{cmH}_{2} \mathrm{O}\right)$ & $188.1 \pm 24.4$ & $169.4 \pm 20.3^{*}$ & $170.2 \pm 23.1$ & $178.3 \pm 29.8$ \\
PImax & $\left(\mathrm{cmH}_{2} \mathrm{O}\right)$ & $102.6 \pm 30.0$ & $96.4 \pm 28.1$ & $100.7 \pm 33.2$ & $105.5 \pm 34.3$ \\
\hline
\end{tabular}

Values are expressed as mean $\pm \mathrm{SD}$. FVC: forced vital capacity; $\mathrm{FEV}_{1}$ : forced expiratory volume;

$\mathrm{FEV}_{1.0 \%}$ : percent of forced expiratory volume; PEmax: maximal inspiratory pressure; PImax: maximal expiratory pressure.

*: comparison with baseline $(\mathrm{p}<0.05)$.

that expiratory muscle activity during exercise not only increases respiratory frequency but also reduces end expiratory lung volume, thereby increasing respiratory efficiency during inspiration, and that increased expiratory muscle activity causes the diaphragm to be pushed further upward, promoting contraction of the diaphragm ${ }^{2,13,14)}$. The abdominal muscle group, a major expiratory muscle, functions to maintain stable posture and control ventilation. During cycling exercise, it appears to serve more as a ventilation controlling muscle than as a posture stabilizing muscle ${ }^{1)}$. These findings suggest that expiratory muscles, although more prone to fatigue than inspiratory muscles, significantly contribute to the ventilation response during exercise.

The observation that no change from baseline was observed in post-exercise PImax while a significant decrease was observed in PEmax also indicates that expiratory muscles are more prone to fatigue than inspiratory muscles following exercise. Previous studies have revealed that inspiratory muscle fatigue stimulates muscle sympathetic nerve activity and reduces blood flow in prime mover muscles ${ }^{15,16)}$. Thus, fatigue of the respiratory muscles may reduce blood flow in the lower extremities and prevent circulation to prime mover muscles during exercise, thereby accelerating muscular fatigue. In this way, fatigue of the expiratory muscles during exercise would affect exercise performance. No decrease from baseline was observed in inspiratory muscle strength measured immediately after exercise. Romer et al. 17) showed that adding positive pressure to inspired air during exercise to reduce the burden on inspiratory muscles did not improve exercise performance and suggested that factors affecting fatigue in the diaphragm, an inspiratory muscle, include not only exercise intensity but also exercise time $^{18)}$. In the present study, inspiratory muscle fatigue was not observed even after high-intensity exercise, which was probably because the exercise time was relatively short. Taken together, expiratory muscle fatigue during ramp load exercise might have affected exercise performance.

Respiratory muscle strength was correlated to maximal load and maximal oxygen uptake, but not to maximal breathing capacity. This might have been attributable to individual differences in respiration pattern at maximal exercise load, such as hyperventilation and straining. In addition, the observation that PEmax was decreased after exercise but $\mathrm{FEV}_{1.0 \%}$ was increased suggests that the exercise-induced increase in sympathetic nerve activity continued even after exercise was completed, resulting in dilatation and reduced resistance in the respiratory tract. However, these hypotheses cannot be confirmed by the findings obtained in the present study, and need to be addressed in future studies.

The above findings indicate a correlation between expiratory muscle strength and exercise performance during ramp load exercise and suggest that respiratory muscle strength is an important factor for the exertion and maintenance of exercise performance during short-term, high-intensity competitive sports, such as short-distance sprint. 


\section{REFERENCES}

1) Abraham KA, Feingold H, Fuller DD, et al. : Respiratory- related activation of human abdominal muscles during exercise. J Physiol, 2002, 541(2): 653663.

2) Henke KG, Sharratt M, Pegelow D, et al. : Regulation of end- expiratory lung volume during exercise. J Appl Physiol, 1988, 64(1): 135-146.

3) Strohl KP, Mead J, Banzett RB, et al. : Regional differences in abdominal muscle activity during various maneuvers in humans. J Appl Physiol, 1981, 51(6): 1471-1476.

4) Laude EA, Duffy NC, Baveystock C, et al. : The effect of helium and oxygen on exercise performance in chronic obstructive pulmonary disease: a randomized crossover trial. Am J Respir Crit Care Med, 2006, 173(8): 865-870.

5) Richardson RS, Sheldon J, Poole DC, et al. : Evidence of skeletal muscle metabolic reserve during whole body exercise in patients with chronic obstructive pulmonary disease. Am J Respir Crit Care Med, 1999, 159(3): 881-885.

6) Mancini D, Donchez L, Levine S: Acute unloading of the work of breathing extends exercise duration in patients with heart failure. J Am Coll Cardiol, 1997, 29(3): 590-596.

7) Verges S, Sager Y, Erni C, et al. : Expiratory muscle fatigue impairs exercise performance. Eur J Appl Physiol, 2007, 101 (2): 225- 232.

8) Gandevia SC: Spinal and supraspinal factors in human muscle fatigue. Physiol Rev, 2001, 81(4): 1725-1789.

9) Kayser B: Exercise starts and ends in the brain. Eur J Appl Physiol, 2003, 90(3-4): 411-419.
10) Dempsey JA, Romer L, Rodman J, et al. : Consequences of exercise-induced respiratory muscle work. Respir Physiol Neurobiol, 2006, 151(2-3): 242250.

11) Lieberman DA, Faulkner JA, Craig AB, et al. : Performance and histochemical composition of guinea pig and human diaphragm. J Appl Physiol, 1973, 34(2): 233-237.

12) Uribe JM, Stump CS, Tipton CM, et al. : Influence of exercise training on the oxidative capacity of rat abdominal muscles. Respir Physiol, 1992, 88(1-2): 171-180.

13) Martin JG, De Troyer A: The behaviour of the abdominal muscles during inspiratory mechanical loading. Respir Physiol, 1982, 50(1): 63-73.

14) Verges S, Schulz C, Perret C, et al. : Impaired abdominal muscle contractility after high-intensity exhaustive exercise assessed by magnetic stimulation. Muscle Nerve, 2006, 34(4): 423-430.

15) St Croix CM, Morgan BJ, Wetter TJ, et al. : Fatiguing inspiratory muscle work causes reflex sympathetic activation in humans. J Physiol, 2000, 529(2): 493504.

16) Sheel AW, Derchak PA, Morgan BJ, et al. : Fatiguing inspiratory muscle work causes reflex reduction in resting leg blood flow in humans. J Physiol, 2001, 537(1): 277-289.

17) Romer LM, Miller JD, Haverkamp HC, et al. : Inspiratory muscles do not limit maximal incremental exercise performance in healthy subjects. Respir Physiol Neurobiol, 2007, 156(3): 353-361.

18) Romer LM, Polkey MI: Exercise-induced respiratory muscle fatigue: implications for performance. J Appl Physiol, 2008, 104(3): 879-888. 